\title{
Deficiência de ferro em obesos acompanhados ambulatorialmente no pré-operatório de cirurgia bariátrica
}

\author{
Iron deficiency in obese patients attending an ambulatory facility for preoperative bariatric surgery
}

DOI: $10.37111 /$ braspenj.2020351015

Ítalo José Lemos de Oliveira

Raquel de Arruda Campos Benjamim

Thayanne Breckenfeld Meneses'

Ana Jessyca da Silva Costa'

Jarson Pedro da Costa Pereira

Amanda Braz do Lago'

Jerluce Alvares Ferraz ${ }^{2}$

Maria Goretti Pessoa de Araújo Burgos

\section{Unitermos:}

Ferritinas. Obesidade. Inflamação.

\section{Keywords:}

Ferritins. Obesity. Inflammation.

\section{Endereço para correspondência:}

Ítalo José Lemos de Oliveira

Av. Prof. Moraes Rego, 1235 - Cidade Universitária

- Recife, PE, Brasil - CEP 50670-901

E-mail: italollemos@hotmail.com

\section{Submissão}

27 de setembro de 2019

Aceito para publicação

12 de fevereiro de 2020

\section{RESUMO}

Introdução: A obesidade é uma crise de saúde global. A cirurgia bariátrica oferece tratamento que pode reduzir o peso, induzir a remissão de doenças relacionadas à obesidade e melhorar a qualidade de vida. Carências nutricionais podem ter sido instaladas antes mesmo da cirurgia, e a anemia pré-operatória é um fator de risco independente. Assim, o presente trabalho tem como objetivo avaliar a deficiência de ferro em pacientes obesos candidatos à cirurgia bariátrica, atendidos no ambulatório de Nutrição de um Hospital Universitário em Recife/Brasil. Método: Estudo transversal, descritivo. Os pacientes foram avaliados quanto a sexo, idade, peso, índice de massa corporal (IMC), dosagens de ferro e ferritina. A deficiência de ferro foi classificada por níveis $<50$ $\mathrm{Ug} / \mathrm{dl} \mathrm{e}<65 \mathrm{Ug} / \mathrm{dl}$ para mulheres e homens, respectivamente. Os níveis de ferritina classificados como deficientes foram aqueles menores que $15 \mathrm{ng} / \mathrm{ml}$, para ambos os sexos. A coleta de dados foi realizada no período de maio de 2017 a junho de 2018, sendo utilizados dados das fichas de acompanhamento nutricional do serviço. Os dados foram tabulados e analisados no software Microsoft Excel versão 10. Resultados: Foram incluídos 75 pacientes adultos, com prevalência do sexo feminino (85,3\%), IMC médio de 47,99 $\pm 7,56 \mathrm{~kg} / \mathrm{m}^{2}$. As médias de ferro sérico $(\mathrm{n}=70) \mathrm{e}$ ferritina $(\mathrm{n}=60)$ foram $76,33 \pm 37,06 \mathrm{Ug} / \mathrm{dl}$ e $160,82 \pm 159,43 \mathrm{ng} / \mathrm{ml}$, respectivamente. Houve elevada prevalência de déficit de ferro sérico $(62,9 \%)$, a ferritina, no entanto, apresentou-se em déficit em apenas 1,7\% da população em estudo. Conclusão: Este estudo revelou presença importante de deficiência de ferro no grupo de pacientes obesos avaliados, sendo a sensibilidade maior detectada por meio do ferro sérico. A avaliação bioquímica do paciente que será submetido ao procedimento é importante, de modo a evitar os agravos à sua saúde e complicações no pós-operatório, auxiliando, assim, na sua recuperação.

\section{ABSTRACT}

Introduction: Obesity is an internationally health crisis. The bariatric surgery offers treatment to reduce body weight, induce remission of obesity-related diseases and improve quality of life. Nutritional deficiencies may have been installed even before surgery, and preoperative anemia is an independent risk factor. Thus, the present study aims to evaluate iron deficiency in obese candidates for bariatric surgery, attending a nutrition ambulatory of a university hospital in Recife/ Brazil. Methods: Cross-sectional, descriptive study. The patients were evaluated according to sex, age, weight, body mass index (BMI), iron and ferritin levels. Iron deficiency was classified by levels $<50 \mathrm{Ug} / \mathrm{dl}$ and $<65 \mathrm{Ug} / \mathrm{dl}$ for women and men, respectively. The ferritin levels classified as deficient were those below $15 \mathrm{ng} / \mathrm{ml}$ for both sexes. Data collection was performed from May 2017 to June 2018, using data from nutrition setting's monitoring forms. Data were tabulated and analyzed using Microsoft Excel software version 10. Results: 75 patients were included, with prevalence of women $(85.3 \%)$, mean BMl of $47.99 \pm 7.56 \mathrm{~kg} / \mathrm{m}^{2}$. The means of serum iron $(\mathrm{n}=70)$ and ferritin $(\mathrm{n}=60)$ were $76.33 \pm 37.06 \mathrm{Ug} / \mathrm{dl}$ and $160.82 \pm 159.43 \mathrm{ng} / \mathrm{ml}$, respectively. There was a high prevalence of serum iron deficiency (62.9\%); however, ferritin was deficient in only $1.7 \%$ of the study population. Conclusion: This study observed an important presence of iron deficiency in the group of obese patients evaluated, with the greatest sensitivity being detected by means of serum iron. The biochemical evaluation of the patient who will be submitted to the bariatric procedure is important, in order to avoid health issues and postoperative complications, thus helping in their recovery.

1. Nutricionista residente do Programa de Residência em Nutrição Clínica do Hospital das Clínicas da Universidade Federal de Pernambuco, Recife, PE, Brasil.

2. Nutricionista preceptora do Ambulatório de Nutrição do Hospital das Clínicas da Universidade Federal de Pernambuco, Recife, PE, Brasil.

3. Professora adjunto do departamento de Nutrição da Universidade Federal de Pernambuco, Recife, PE, Brasil. 


\section{INTRODUÇ̃̃O}

Segundo a Organização Mundial de Saúde (OMS)', a presença de anemia e sua gravidade são definidas de acordo com os limiares de hemoglobina e outros parâmetros hematológicos e induz consequências à saúde, mesmo quando a anemia não é clinicamente aparente. Cerca de $90 \%$ das anemias estão relacionadas à deficiência de ferro, um nutriente considerado essencial, que age principalmente na síntese de glóbulos vermelhos e no transporte de oxigênio para demais células do corpo².

O ferro utilizado pelo organismo é proveniente da dieta e reciclagens de hemácias remanescentes ${ }^{2}$. A absorção de ferro é regulada pelo hormônio peptídico hepático, a hepcidina, que também controla a liberação de ferro das células que reciclam ou armazenam ferro, regulando, assim, as concentrações plasmáticas do nutriente ${ }^{3}$. Estudos indicam que a hepcidina é o regulador negativo predominante da absorção intestinal de ferro e sua síntese é estimulada pela sobrecarga de ferro e pelos processos inflamatórios, que podem estar aumentadas em até 100 vezes na anemia por inflamação ${ }^{4}$.

A obesidade é reconhecida como uma crise de saúde global e suas taxas estão aumentando, com pelo menos 300 milhões de pessoas em todo o mundo agora classificadas como obesas 5 . No Brasil, esses índices aumentaram nos últimos 25 anos; de acordo com o Ministério da Saúde, através da Vigilância de Fatores de Risco e Proteção para Doenças Crônicas por Inquérito Telefônico (VIGITEL), cerca de $18,9 \%$ da população brasileira encontram-se obesa ${ }^{6}$.

Na obesidade, têm-se verificado cada vez mais a prevalência de carências nutricionais que podem ter sido instaladas antes mesmo da cirurgia ${ }^{3}$. A anemia pré-operatória afeta $30 \%$ a $40 \%$ dos pacientes submetidos a cirurgias de grande porte e é um fator de risco independente para transfusão sanguínea, morbidade e mortalidade perioperatórias ${ }^{1}$.

A obesidade correlaciona-se com o aumento das atividades inflamatórias, que libera citocinas inflamatórias pelas adipocinas, situadas nos adipócitos, como interleucina 6, fator de necrose tumoral alfa, proteína $C$ reativa e outras. Assim, a inflamação provocada pela obesidade, configura o indivíduo a um estado inflamatório de baixa intensidade?

A cirurgia bariátrica oferece um tratamento que pode reduzir o peso, induzir a remissão de doenças relacionadas à obesidade e melhorar a qualidade de vida ${ }^{5}$. Dentre as técnicas cirúrgicas mais utilizadas, destaca-se a gastroplastia com derivação intestinal (by-pass). Trata-se de uma técnica mista, com predominância do fator restritivo, o que pode contribuir para deficiência de nutrientes ${ }^{8}$.

As alterações fisiológicas e anatômicas que ocorrem decorrentes da cirurgia bariátrica resultam em deficiências macro e micronutricionais, especialmente a deficiência de ferro, o que pode ter seu agravamento no pós-operatório ${ }^{8,9}$.
A anemia pós-operatória é ainda mais comum (até 80-90\%) devido à perda de sangue associada à cirurgia, redução da eritropoiese induzida por inflamação e/ou anemia preexistente'.

Dessa forma, o presente trabalho tem como objetivo avaliar a deficiência de ferro em pacientes obesos candidatos à cirurgia bariátrica, atendidos no ambulatório de nutrição de um hospital universitário em Recife.

\section{MÉTODO}

Estudo transversal, de caráter descritivo, conduzido no ambulatório de Nutrição pré-bariátrica do Hospital das Clínicas da Universidade Federal de Pernambuco (HC/UFPE). A coleta de dados compreendeu informações registradas nas fichas de acompanhamento ambulatorial do setor de Nutrição do hospital, referentes aos pacientes atendidos no período de maio de 2017 a junho de 2018.

Foram incluídos pacientes com índice de massa corporal (IMC) $\geq 30 \mathrm{~kg} / \mathrm{m}^{2}$, idade $\geq 20$ anos e que apresentassem exames de ferro sérico ou ferritina. Foram coletados dados referentes a sexo e idade, para caracterização da amostra, além de peso e altura, para cálculo do IMC, e resultados dos exames de ferro e ferritina.

A deficiência de ferro foi considerada quando os níveis foram $<50 \mathrm{Ug} / \mathrm{dl}$ e $<65 \mathrm{Ug} / \mathrm{dl}$ para mulheres e homens, respectivamente. Os níveis de ferritina classificados como deficientes foram aqueles menores que $15 \mathrm{ng} / \mathrm{ml}$, para ambos os sexos. Foram considerados insuficiente ativos ou sedentários aqueles pacientes que realizaram $<150$ minutos/ semana de atividade física.

O protocolo de pesquisa foi pautado pelas normas éticas para pesquisa envolvendo seres humanos, constantes na resolução $N^{\circ}$ 466/12 do Conselho Nacional de Saúde, e foi submetido a avaliação do Comitê de Ética e Pesquisa da instituição, sendo aprovado com número do parecer 2.877.855. Os dados foram tabulados e analisados no software Microsoft Excel versão 10.

\section{RESULTADOS}

A amostra foi composta por 75 pacientes adultos, com prevalência do sexo feminino (85,3\%), com média de idade de 37,44 $\pm 10,23$ anos. A média de peso foi de $123,9 \pm 24,9 \mathrm{~kg}$, IMC médio de 47,99 $\pm 7,56 \mathrm{~kg} / \mathrm{m}^{2}$, sendo $61,3 \%$ dos pacientes classificados com obesidade entre os graus I, II e III e 38,7\% com super- e supersuperobesidade (IMC $\left.>50 \mathrm{~kg} / \mathrm{m}^{2}\right)$. As médias de ferro sérico $(n=70)$ e ferritina $(n=60)$ foram $76,33 \pm 37,06 \mathrm{Ug} / \mathrm{dl}$ e $160,82 \pm 159,43 \mathrm{ng} / \mathrm{ml}$, respectivamente. Houve elevada prevalência de déficit de ferro sérico $(62,9 \%)$ na população estudada (Tabela 1). 


\begin{tabular}{|c|c|c|}
\hline Variável & $\mathrm{n}$ & $\%$ \\
\hline \multicolumn{3}{|l|}{ Índice de massa corporal } \\
\hline $\mathrm{IMC}$ entre $30-50 \mathrm{~kg} / \mathrm{m}^{2}$ & 46 & 61,3 \\
\hline $\mathrm{IMC}>50 \mathrm{~kg} / \mathrm{m}^{2}$ & 29 & 38,7 \\
\hline \multicolumn{3}{|l|}{ Sexo } \\
\hline Masculino & 11 & 14,7 \\
\hline Feminino & 64 & 85,3 \\
\hline \multicolumn{3}{|l|}{ Ferro sérico } \\
\hline Normal & 44 & 62,9 \\
\hline Déficit & 26 & 37,1 \\
\hline \multicolumn{3}{|l|}{ Ferritina } \\
\hline Normal & 59 & 98,3 \\
\hline Déficit & 1 & 1,7 \\
\hline
\end{tabular}

\section{DISCUSSÃO}

A amostra foi predominantemente composta por indivíduos do sexo feminino, corroborando com outros estudos realizados na população de pessoas submetidas a cirurgia bariátrica, como no trabalho de Cazzo et al. ${ }^{10}$, em que $70 \%$ dos indivíduos eram mulheres. Corroborando, então, por consequência, com dados publicados pelo Ministério da Saúde, que revelam que 13\% dos adultos brasileiros são obesos e $43,3 \%$ possuem sobrepeso corporal, sendo o índice entre as mulheres $(13,6 \%)$ maior do que dos homens $(12,4 \%)$, - que poderia justificar uma possível maior demanda de mulheres que se submetem aos procedimentos cirúrgicos como estratégia de reversão da obesidade ${ }^{8}$.

Na população estudada, foi possível observar, também, que os pacientes apresentavam deficiência de ferro sérico, indicando um possível quadro de anemia ferropênica, tendo importante relevância clínica, pois sabe-se que tal micronutriente e responsável por diversos processos fisiológicos e metabólicos, sendo essencial para o funcionamento vital do organismo. A ingestão inadequada e/ou a má absorção são duas das principais causas que promovem a ferropenia e estão diretamente relacionadas às alterações mecânicas da cirurgia bariátrica, indicando a necessidade de verificação dos níveis bioquímicos, tal como sinais e sintomas clínicos que indiquem a deficiência deste mineral para a realização de possível intervenção dietética e medicamentosa, se necessário ${ }^{11-14}$.

Estudos discutem a coexistência antagônica das duas realidades, onde se discute uma situação carencial (anemia ferropriva) e uma condição que rotineiramente e superficialmente define-se pelo excesso alimentar (obesidade), indicando que esta condição, na maioria das vezes, não está associada à ingestão excedente de nutrientes, apresentando, por sua vez, uma interface contrária a este argumento. Assim, é facilmente compreendida a existência da situação da obesidade associada à anemia ferropriva, que perpassa por condições não somente metabólicas e patológicas, como os mecanismos de absorção do ferro, mas também com situações de vulnerabilidade social, onde alguns indivíduos - o que inclusive se observa na realidade da população estudada na presente pesquisa - não possuem acesso contínuo aos alimentos fonte que possuem melhor biodisponibilidade do ferro-heme, como é o caso das carnes vermelhas ${ }^{15}$. Foi observado, no presente estudo, que a ferropenia não apresentou prevalência elevada, o que pode figurar uma possibilidade de sucesso no momento e tratamento nutricional pós-operatório. É conhecido que uma das principais consequências da ressecção gástrica é a diminuição dos níveis séricos de ferro, que pode ocorrer principalmente por três causas: baixa ingestão de ferro oral, por intolerância aos alimentos ricos em ferro; exclusão da porção duodenal do intestino delgado, nos casos das cirurgias de by-pass gástrico, onde o duodeno releva-se por ser o principal sítio de absorção do ferro, o que também promove uma restrição na ação das enzimas pancreáticas que são liberadas no mesmo sítio, e estas auxiliam o processo de liberação do ferro ligado ao heme; e, por fim, pode ocorrer pela diminuição da acidez gástrica ocasionada por um menor número de células parietais, o que dificulta a conversão do ferro férrico para a sua forma ferrosa ${ }^{15,16}$.

Os estudos que envolvem avaliação bioquímica e hematológica no paciente em perioperatório da cirurgia bariátrica normalmente observam que, além da ferropenia, pode existir comprometimento metabólico e nutricional, que envolve outras vitaminas e minerais, como vitamina B12, ácido fólico, influenciando também na quantidade das proteínas totais e suas frações, como a albumina e transferrina ${ }^{17}$.

Os mesmos efeitos, impactos nutricionais e metabólicos supramencionados também se aplicam aos valores séricos da ferritina, que é normalmente conhecida por armazenar os estoques de ferro do organismo, que, por sua vez, em decorrência de toda complexidade já discutida do período perioperatório da cirurgia bariátrica, naturalmente tende a apresentar diminuição nos valores hematológicos, como observado em estudos $^{17}$. No entanto, no presente estudo, foi observado um panorama distinto, onde os indivíduos apresentaram apenas diminuição do ferro sérico, sem comprometimento nos valores laboratoriais da ferritina e, em alguns indivíduos, esses níveis de ferritina até se demonstraram mais elevados.

Esses valores da ferritina sérica precisam ser cautelosamente interpretados, pois existe o viés bioquímico, o que não fornece segurança ao estudo para determinar se os estoques de ferro do organismo estão adequados. Por se tratar de uma proteína de fase aguda positiva, esta pode apresentar seus níveis séricos elevados em situações de processo inflamatório, 
- que conflui com a realidade da população, uma vez que a obesidade configura um processo inflamatório crônico e com eventos agudos. Seus valores podem ser influenciados por processo infeccioso e idade do indivíduo, sendo então necessário realizar outros processos diagnósticos, até mesmos clínicos, para excluir ou confirmar a ocorrência de um processo infeccioso ${ }^{17}$.

Valores semelhantes foram encontrados em estudo realizado por Angeli et al. ${ }^{18}$, demonstrando que, mesmo em situação de ferropenia, alguns indivíduos podem apresentar valores séricos de ferritina normais e/ou elevados. Assim, reforça-se a ideia de que é importante fazer uma avaliação completa do perfil e cinética do ferro, pois usar um dado bioquímico isoladamente pode promover um viés de confusão no momento da interpretação, podendo gerar condutas e intervenções inapropriadas para a realidade do paciente candidato à cirurgia bariátrica.

\section{CONCLUSÃO}

Este estudo observou revelou presença importante de deficiência de ferro no grupo de pacientes obesos avaliados, sendo a sensibilidade maior detectada por meio do ferro sérico. As carências nutricionais têm elevada prevalência na obesidade, podendo ser exacerbadas no pós-operatório da cirurgia bariátrica. Observou-se, também, que a maioria dos pacientes apresentava níveis normais de ferritina, porém vale ressaltar que estes valores devem estar na mediana da referência, tendo em vista o perfil da população em estudo ser, em sua maioria, mulheres em idade fértil, sendo necessário, assim, um olhar crítico para os resultados encontrados. Desta forma, faz-se necessária uma avaliação bioquímica em valores otimizados e tratamento prévio do paciente que será submetido ao procedimento, de modo a evitar os agravos à sua saúde e complicações no pós-operatório, auxiliando, assim, na sua recuperação.

\section{REFERÊNCIAS}

1. Gómez-Ramírez S, Bisbe E, Shander A, Spahn DR, Muñoz M. Management of perioperative iron deficiency anemia. Acta Haematol [Internet]. 2019;142(1):21-9.

2. Aragão CAP, Araújo MSM. Prevalência de anemia ferropriva em obesos em preparo para cirurgia bariátrica. Comun Ciênc Saúde. 2015;26(3/4):145-52.

3. Sangkhae V, Nemeth E. Regulation of the iron homeostatic hormone hepcidin. Adv Nutr. 2017;8(1):126-36.
4. Braga JAP, Amâncio OMS, Vitalle MLL. O ferro e a saúde das populações. São Paulo: Roca;2006.

5. Neff KJ, Olbers T, le Roux CW. Bariatric surgery: the challenges with candidate selection, individualizing treatment and clinical outcomes. BMC Med [Internet]. 2013;11(1):8.

6. Brasil. Ministério da Saúde. Vigitel Brasil 2016 Saúde Suplementar: vigilância de fatores de risco e proteção para doenças crônicas por inquérito telefônico [recurso eletrônico] Agência Nacional de Saúde Suplementar. Brasília: Ministério da Saúde; 2017.

7. Mascarenhas PTCMC. Efeito do consumo da polpa de açaí (Euterpe oleracea Mart.) sobre as concentrações de adipocinas, medidas antropométricas, de composição corporal, parâmetros bioquímicos, clínicos e dietéticos em mulheres eutróficas e com excesso de peso aparentemente saudáveis. [Dissertação de Mestrado em Saúde e Nutrição]. Ouro Preto: Universidade Federal de Ouro Preto; 2015. 106p.

8. Carvalho AS, Rosa RS. Cirurgias bariátricas realizadas pelo Sistema Único de Saúde no período 2010-2016: estudo descritivo das hospitalizações no Brasil. Epidemiol Serv Saúde. 2019;28(1):e2018260.

9. Enani G, Bilgic E, Lebedeva E, Delisle M, Vergis A, Hardy $\mathrm{K}$. The incidence of iron deficiency anemia post-Roux-en-Y gastric bypass and sleeve gastrectomy: a systematic review. Surg Endosc. 2019. doi: 10.1007/s00464-019-07092-3. [cited 2020 Sep 12]. Available from: http://link.springer.com/10.1007/ s00464-019-07092-3

10. Cazzo E, Valerini FG, Chaim FHM, Soares PFC, Ramos AC, Chaim EA. Early weight loss outcomes and glucose metabolism parameters after banded versus non-banded one anastomosis gastric bypass: a prospective randomized trial. Arq Gastroenterol. 2019;56(1):15-21.

11. Costa MAP, Vasconcelos AGG, Fonseca MJM. Prevalência de obesidade, excesso de peso e obesidade abdominal e associação com prática de atividade física em uma universidade federal. Rev Bras Epidemiol. 2014;17(2):421-36.

12. World Health Organization. Vitamin and mineral requirements in human nutrition: report of a joint FAO/WHO expert consultation. Bangkok: World Health Organization; 1998.

13. Frazer DM, Anderson GJ. Iron Imports I. Intestinal iron absorption and its regulation. Am J Physiol Gastrointest Liver Physiol. 2005;289(4):G631-5.

14. Buchwald H. Overview of bariatric surgery. J Am Coll Surg. 2002;194(3):367-75.

15. Batista Filho M, Souza AI, Miglioli TC, Santos MC. Anemia e obesidade: um paradoxo da transição nutricional brasileira. Cad Saúde Pública. 2008;24(suppl. 2):247-57.

16. Traina F. Deficiência de ferro no paciente submetido à ressecção gástrica ou intestinal: prevalência, causas, repercussões clínicas, abordagem diagnóstica e prevenção. Rev Bras Hematol Hemoter. 2010;32(supl. 2):78-83.

17. Santos CF, Amado T, Sandri YP, Frizzo MN. Alterações dos parâmetros laboratoriais em pacientes submetidos à cirurgia bariátrica. Rev Saúde Integ. 2014;7(13-14):9-30.

18. Angeli LA, Falqueto LE, Perim LP, Lorenzoni GD, Santos MAR, Guzzo MF. Avaliação nutricional pré-operatória de pacientes obesos acompanhados em hospital de referência do SUS. Rev Bras Pesq Saúde. 2014;16(supl):5-9.

Local de realização do estudo:Ambulatório de Nutrição do Hospital das Clínicas da Universidade Federal de Pernambuco, Recife, PE, Brasil.

Conflito de interesse: Os autores declaram não haver.

Trabalho apresentado no $23^{\circ}$ Congresso Brasileiro de Nutrição Parenteral e Enteral, realizado em Foz do Iguaçu, PR, Brasil, em 21 de outubro de 2019. 